\title{
Marketing Strategy Analysis of Green Agricultural products -- Taking Daqing of Heilongjiang Province as an Example
}

\author{
Xueli Yang \\ Heilongjiang Bayi Agricultural University, Daqing, Heilongjiang, China
}

Key words: Green Agricultural Products, Marketing, Marketing Strategy

Abstract: Nowadays, food safety has been paid close attention to. People's consumption demand for safe and green agricultural products is constantly increasing. With the increasing green consciousness of consumers, green agricultural products will become an indispensable part of human pursuit of healthy life, which is the inevitable trend of development. In recent years, the status of agricultural products has been greatly improved, but there are still some problems to be improved in the marketing of urban agricultural products, such as the limitation of target customers, the lack of brand building, the weak marketing strength and the low market recognition. Based on the analysis of the consumption intention and purchase behavior of urban residents for green agricultural products, and on the basis of the analysis of typical foreign development models, this paper explores and puts forward marketing strategies to break through the development dilemma of green agricultural products, strengthen the brand construction of green agricultural products, optimize the logistics distribution and circulation system, and promote the construction of green agricultural products traceability platform with big data.

\section{Introduction}

With the deepening of the concept of green development and the improvement of living standards, urban citizens continue to pursue high-quality life. The demand structure for food has changed, and the demand structure for agricultural products is also changing. Meanwhile, the demand for green agricultural products is increasing. Green agricultural products and green marketing are the products of the development of the times. The certification of green agricultural products is divided into pollution-free agricultural products, green agricultural products and organic agricultural products. The certification conditions of these three levels of agricultural products and food are gradually increased. Green food is a non polluting, safe, high-quality and nutritious food that follows the principle of sustainable development and is approved by the "China Green Food Development Center" under the Ministry of agriculture, which is produced in a specific way and licensed to use green signs. Green agricultural products are ecological, safe and high-quality agricultural products. They are green food produced in specific ways and conditions. Green 
marketing advocates ecological security, which is reflected in the green marketing process, and no pollution in the production, consumption and recycling of enterprises [1]. Green marketing can realize the unity of agricultural economic benefits and sustainable development of agriculture. It also meets the needs of consumers' agricultural products and the agricultural environment, realizing the green management of agricultural products by enterprises in the whole process. The planting, production, sales and after-sales of agricultural products are all based on the premise of maintaining the ecological environment. The value and significance of green marketing of agricultural products are embodied in conforming to the relationship between agricultural economic benefits and ecological natural environment, realizing the goal of harmonious coexistence and sustainable development of human and nature. In this context, this study starts from green agricultural products, ensuring the quality and safety of agricultural products to meet the needs of consumers. The study tries to explore a reasonable path to carry out the whole green model, explore the combination of modern information technology and agricultural development, and explore the marketing strategies and suggestions of green agricultural products suitable for Daqing city.

\section{Development background and marketing status of green agricultural products in Daqing}

\subsection{Development background of green agricultural products in Daqing}

Daqing city is known as the "city of natural lakes" for its vertical and horizontal lakes and relatively superior natural conditions. The black soil is fertile, with hot and rainy seasons, which is conducive to the growth of crops. In recent years, the cultivation industry has been improved in an all-round way, and modern agriculture has been developed relying on Daqing national modern agricultural demonstration zone. In 2019, the government work report said the construction of an agricultural "three reduction" demonstration base is 330,000 acre, and that a green food production base is 400,000 acre. The cultivation of high-quality soybeans, rice and corn is 165,000 acre, 330,000 acre and 1,000,000 acre separately. The total grain output has remained stable, reaching more than 5 billion kg. With the development of Daqing economy, the living standard of the residents is improved. The per capita disposable income of the residents is on the rise. The Engel coefficient is on the decline and the consumption is upgrading. People are more and more pursuing high-quality life. They also more and more paying attention to health. Green agricultural products conform to the development of the times, becoming the embodiment of people's advocating for nature and recognition of green development, and the consumption of green agricultural products is also in line with the consumption Trend development.

\subsection{Actively practise the marketing mode of "Internet + Agricultural" products.}

Since the State Council issued the guiding opinions on actively promoting the "Internet + " action, Daqing city has actively responded to the implementation and the promotion of the "Internet + Agriculture”. The agricultural product industry has gradually become information-based and technological. Under the background of "Internet + Agriculture", it has innovated the marketing mode of agricultural products, making full use of the advanced technology of the Internet, and relying on the e-commerce platform to package agricultural products sale. It is essential to seize the characteristics of sharing and openness of the Internet to quickly occupy the market and help the marketing of agricultural products. Thanks to the Internet technology, agricultural products online 
marketing is from traditional e-commerce to mobile e-commerce, which has the characteristic of the portability and easy operation and is not affected by geographical location, attracting more and more enterprises and consumers to participate in. In the development of agricultural products industry, we should follow the concept of quality and green agriculture, and pay attention to promoting the development of green agricultural products industry. The marketing mode of "Internet +" has rapidly met the demand of consumers for green agricultural products, improved the sales efficiency of green agricultural products, and promote the development of agricultural economy. Some green agricultural products enterprises start from the needs of consumers, and realize the "family personalized customization", distributing different agricultural products according to the needs of families, which is deeply loved by consumers. The promotion of green agricultural products also promotes the transformation and upgrading of agriculture, the transformation of farmers' management, and the development of ecological agriculture. It promotes competitiveness of agricultural products. It also uses Internet technology to promote the operation and sales of green agricultural products, expanding marketing channels of green agricultural products.

\section{Problems in the marketing of green agricultural products}

\subsection{The concept of green marketing of agricultural enterprises is limited}

The premise of green marketing is green production. Green agricultural products generally grow in rural areas with better ecological environment. Because of its relatively superior natural conditions, pollution-free green planting methods are used in the planting process. High input and low output are the characteristics of green agricultural products. Driven by the interests of farmers, some traditional farmers still can not get rid of the temptation of profits, and even give up the standards of food quality and safety. In order to improve the output and increase the income, chemical dosage is used in the planting process, regardless of the damage to the soil and the pollution of the environment. Agricultural products, especially fruits and vegetables, are no longer green and can not meet the standard of green organic. In the long run, it will inevitably cause the aversion of the consumer groups, easily make consumers feel cheated, cause consumers' worries and doubts about the "green" degree, and inevitably affect the purchase desire, purchasing power and consumer selection Choice and preference. At present, the traditional sales channels of green agricultural products are mainly concentrated in the agricultural trade market, shopping malls, supermarkets, and wholesale markets, etc., sharing channels with ordinary agricultural products, which results in information asymmetry. Due to the lack of professional knowledge, it is difficult for general consumers to distinguish the appearance of products. It is also difficult to tell whether fresh fruits and vegetables, which are packaged with the word "green", are really green food or have been planted with chemicals. The existence of inferior products and lack of inspection and supervision will lead to the possibility of high-end products being expelled from the market by low-end products.

\subsection{The brand construction of green agricultural products is insufficient}

There are many kinds of green agricultural products in the market, but there are little well-known brands. In recent years, the awareness of brand building of agricultural enterprises in Daqing city 
has been enhanced, but the publicity methods are insufficient, and the guiding role of customer selection is insufficient. On the one hand, it is difficult to achieve premium and income increase; on the other hand, it affects sales volume, hindering production enthusiasm. Some enterprises and farmers do not have a strong awareness of the main body of green agricultural products brand management. They often pay too much attention to the immediate interests and sales volume. Some enterprises, influenced by their own funds and scale, pay attention to the increase of the number of trademark registration and "three products and one standard" certification in brand construction, and neglect the lasting maintenance of the brand. With the enhancement of people's awareness of green health, the focus of consumption of agricultural products will inevitably tends to focus on the direction of brand, and well-known brands can better meet the psychological needs of consumers, so the follow-up development of brand is more important, which is in contradiction with the current phenomenon of less investment in marketing and promotion of green agricultural products brand and imperfect marketing system. In addition, the marketing channels of agricultural products are lack of diversification, and green agricultural products use network marketing, but the sales volume is not stable, the number is not large, and the combination with traditional marketing methods is not close. The marketing channels are not rich. The market outside the local area is lack, and the brand coverage is narrow.

\section{Marketing characteristics of foreign green agricultural products}

\subsection{Japan South Korea model: outstanding channel construction of green agricultural product marketing}

The common characteristics of Japan and South Korea are the scarcity of land, less cultivated land per capita and limited operating cultivated land. Due to the scarcity of agricultural materials in Japan and South Korea, the production of green agricultural products adopts the mode of small-scale decentralized management and the mode of small-scale production and large circulation in product marketing. Although it is decentralized management, it pays attention to channel construction, and constructs a green agricultural product channel construction system integrating the government, enterprises and consumers, which is relatively perfect, forming a typical Japanese and South Korean model[2]. This kind of channel construction system makes the market system developed, the transportation system developed, and the radiation circle of green agricultural products wholesale market increased. On the one hand, relying on the superior infrastructure railway and road, and the advanced logistics supply chain technology, green agricultural products are quickly distributed to the surrounding urban areas; on the other hand, channel construction system makes full use of the developed wholesale market network conditions to achieve the optimization of the layout of green agricultural products. The price center of green agricultural products has been formed, and the market price system of green agricultural products has operated well.

\subsection{EU model: improve efficient logistics and distribution system}

The EU has actively implemented resource recycling. It also implements policies and systems related to environmental protection, and improve the logistics system and the distribution radius starting from the distribution link. We should strengthen the norms of all links of green agricultural 
products, from packaging to transportation, from storage to circulation. The government optimizes the layout and renovates the transportation main roads and storage facilities for the development of green agricultural products industry, so as to realize efficient circulation of green agricultural products. According to statistics, $78 \%$ of green agricultural products distribute from the origin to the distribution center to the retailer, while only about $20 \%$ of them pass through the wholesaler [3]. The channel link has greatly reduced the cost, accelerated the circulation and improved the distribution efficiency, establishing the green agricultural products logistics system in line with the requirements of EU environmental protection, and basically realizing the virtuous circle of resources, ecology and society. The European Union also has and relies on technical consulting companies to classify distribution companies, transportation and storage companies serving green agricultural products to further improve the circulation efficiency of green agricultural products and improve the EU's fast and efficient logistics and distribution system for green agricultural products.

\section{Suggestions on marketing strategies of urban green agricultural products}

\subsection{Strengthen the brand construction of green agricultural products}

With the continuous enhancement of residents' consumption concept and environmental awareness, our city should vigorously develop brand strategy in the marketing of green agricultural products, stimulate the enthusiasm and initiative of agricultural enterprises and farmers to jointly maintain the brand, and enhance the regional brand awareness. Enhance the competitiveness of green agricultural products, and create private brands based on regional brands to achieve win-win results. As far as the market is concerned, it is becoming a trend to develop brands based on the quality difference of competitive products. Agricultural enterprises need to stick to their own strength to find consumers of characteristic green agricultural products, which is the only way to be safer and more operational. The so-called differentiation is based on consumer demand perception. The differences in production base, $\mathrm{R} \& \mathrm{D}$ technology and other aspects cannot be effectively reflected in the differences in consumer vision, touch and taste. Fundamentally speaking, this is an invalid difference, which should be paid attention to by agricultural enterprises.

Highlight the brand value of green agricultural products and clear brand positioning. On the one hand, green agricultural products themselves cause less environmental pollution, which is conducive to supporting and protecting the ecological environment and realizing green development. On the other hand, green agricultural products are conducive to realizing consumers' pursuit of healthy life, adapting to the development trend of consumption, and occupying an advantage in the future market. Highlight the positive role of green agricultural products in ecological protection, and guide the general public to protect the ecology and protect their homes. To build a brand and enhance the added value of products, we have achieved double effects, enhanced the awareness of environmental protection and increased purchasing power, making green agricultural products achieve high quality and good price.

Give more culture to agricultural products. Culture is also the spiritual pursuit of consumers, no exception in the field of food. Nowadays, consumers pay more attention to food culture and to the concept behind the products when they consume [4]. Therefore, from the perspective of cultural marketing, we can enrich more cultural connotations of green agricultural products. In terms of brand positioning, we should fully tap the historical and cultural resources of our country, enrich the 
brand of green agricultural products, expand marketing strategies, highlight the image of green agricultural products, and form a "brand impression", so that consumers can consume with this good cultural impression, and enjoy the cultural connotation in spirit while satisfying the material consumption.

\subsection{Optimize logistics distribution and circulation system}

From the basic framework of the circulation of fruits and vegetables agricultural products (as shown in Figure 1), the development and growth of green agricultural products industry depends on the development and improvement of marketing and circulation system to a great extent. Without a perfect commodity circulation system, the development of fruits and vegetables production will be restricted. For the special properties of green fruits and vegetables, a perfect cold chain logistics system should be established to ensure the quality of fruits and vegetables and improve customer satisfaction.

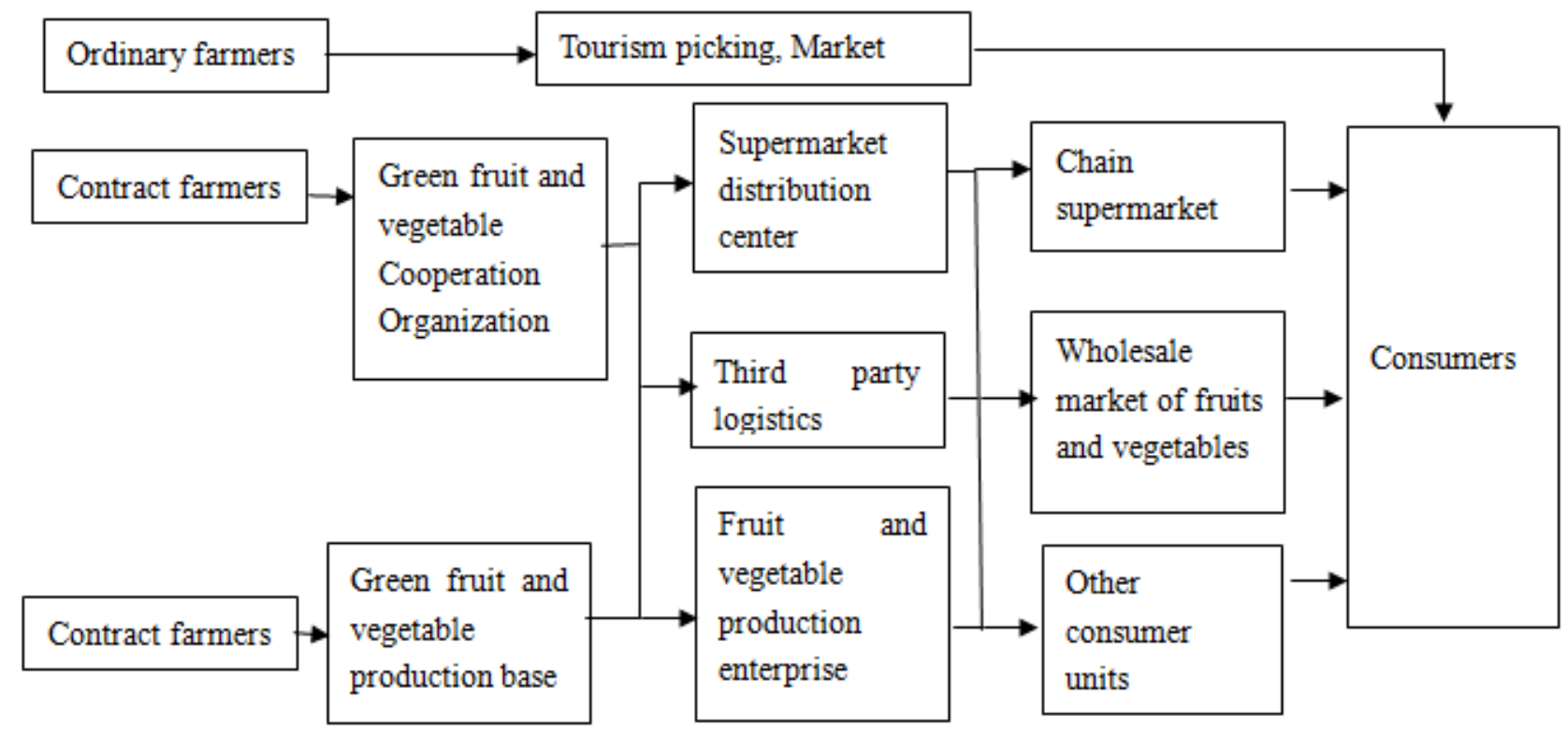

Figure 1: Basic framework of green fruits and vegetables circulation

To integrate small farmers and ordinary farmers into the green fruit and vegetable industry chain, we need to build different levels of fruit and vegetable logistics park and fruit and vegetable trading market. Establish green agricultural products Park and wholesale market, adopt the principle of proximity, so as to save the initial operating cost, and set up perfect refrigeration service facilities. We also should improve the circulation industry chain, improve the circulation speed and efficiency, and build a comprehensive information platform for agricultural products circulation [5]. To minimize the loss of fruits and vegetables in the process of transportation, consumers should be provided with green organic fruits and vegetables products.

\subsection{Big data promotes the construction of green agricultural products traceability platform}

In the context of rural revitalization, advanced technology to optimize the marketing mode of agricultural products is used and marketing channels of agricultural products are innovated to 
revitalize the rural economy. The data platform and the green agricultural products traceability system are used to meet consumers' psychological trust in green safety and enhance consumers' trust in green agricultural products so that purchasing power is also enhanced. Through the green agricultural products traceability system platform, consumers can learn about all aspects of agricultural products from growth to mature processing. For example, in terms of agricultural materials input, the factors such as seeds, fertilizers and pesticides purchased by producers directly affect the quality of agricultural products, which are also the factors of special concern to the green agricultural products consumers. The R \& D and use of the traceability system should be realized through the procurement system Name system and other methods, making the data of agricultural materials purchased and used by green agricultural product growers fully traceable and realize supervision. Consumers are still worrying about the use of ripening agents, colorants and bulking agents in the purchase of green agricultural products. Therefore, a visual supervision system should be implemented in the planting area. The planting of green agricultural products should be operated and managed under an open condition with supervision and record, so as to realize the information display of the growth cycle of green agricultural products, which can be traced all the way Taiwan.

\section{Acknowledgements}

Heilongjiang Bayi Agricultural University Support Program for San Heng San Zong”, Project No.: TDJH201811

\section{Reference}

[1] Darong Y. (2010) Research on marketing of characteristic agricultural products in an innovative society. North economic and trade, (10): 75-77

[2] Jing Z. (2014) Characteristics and reference of foreign green agricultural product marketing. Food and machinery, (5): $276-278$

[3] Xuan W. (2011) Analysis of green marketing. China market, (12): 50-52

[4] Ying W. (2019) Research on brand positioning and marketing strategy optimization of green agricultural products from the perspective of green production. Agricultural economy, (8): 127-129

[5] Meiyu L., Chengmin W. (2019) Research on Optimization of circulation channel mode of fresh agricultural products under the background of "Internet +". Journal of Beijing Jiaotong University (SOCIAL SCIENCE EDITION) (1): 102-114 